\title{
Avaliação da referência e contrarreferência no Programa Saúde da Família na Região Metropolitana do Rio de Janeiro (RJ, Brasil)
}

\author{
Evaluation of reference and counter-reference in the Family $\mathrm{H}$ ealth \\ Program at the M etropolitan Region of Rio de Janeiro (RJ, Brazil)
}

Carlos Gonçalves Serra ${ }^{1}$

Paulo HenriquedeAImeida Rodrigues ${ }^{1}$

1 Universidade Estácio de Sá. Campus Arcos da Lapa. Rua do Riachuelo, 27/60 andar. 20230-010 Rio de Janeiro RJ. carlosgser@gmail.com
Abstract This article presents the results of re search on the functioning of the reference and counter-reference system in support of activities carried out by the Brazilian Family $\mathrm{H}$ ealth Program in the cities of Duque de Caxias (RJ) and Rio de Janeiro. The research had as premise that the resolution capacity of the Brazilian Family Health Program depends on the support of specialist's medical appointments and diagnosis test offered by health care unities of the secondary lev$\mathrm{el}$, and depends upon reference and counter-reference information. The research's results may be summarized as: (1) limited offer of specialized services in the secondary level of the health care system; (2) nonexistence or failure of counter-reference information; (3) deficient organization of regulation activities; (4) insufficient utilization of clinical guidelines for patient referrals; (5) precariousness of information and communication systems; (6) high level of political influence in the management of health care services; and (7) different denominations and great diversity of types of service offered by secondary health care unities. Key words Primary Health Care, Family Health Care, Referenceand counter-reference, $\mathrm{H}$ ealth care networks and systems management
Resumo Este artigo apresenta os resultados da pesquisa realizada sobre o sistema de referência e contrarreferência em apoio ao Programa/Estraté gia Saúde da Família nos municípios de Duque de Caxias (RJ) edo Rio de Janeiro. A pesquisa contou com apoio do Conselho Nacional dePesquisa edas Secretarias M uni cipais deSaúde (SM S) de Duque deCaxias edo Rio deJaneiro. Partiu-seda premissa de que o PSF, para ser resolutivo, precisa contar com o apoio do nível secundário em termos de realização de consultas e exames especializados, indi spensáveis para a conclusão de diagnósticos, 0 quedependedo funcionamento da referência econtrarreferência. Entre os resultados obtidos que dificultam 0 acesso dos pacientes e 0 bom funcionamento dos si stemas de referência e contrarreferência nasáreas pesquisadas, estão: (1) limitada oferta de consultas e exames; (2) inexistência ou precariedade da contrarreferência; (3) má organização das atividades de regulação; (4) baixa utilização de protocol os clínicos para encaminhamentos; (5) precariedade em termos de sistemas de informação e comunicação; (6) significativa influência política na gestão das unidades; (7) grande diversidade na denominação das unidades de saúde e multiplicidade das grades de oferta de serviços. Palavras-chave Atenção Primária deSaúde, Saúde da Família, Referência e contrarreferência, Regulação de redes esistemas de saúde 
Introdução

A saúde da família é considerada como uma das principais estratégias de reorganização do Sistema Único de Saúde (SUS) e da reorientação da atenção primária, ou básica, em particular no quediz respeito ao resgate das diretrizes e princípios do primeiro e das práticas de atenção. Desde a sua criação, em 1994, o Programa Saúde da Família (PSF) experimenta um significativo crescimento em todo o país, com mais de 28 mil equipes implantadas até março de 2008 , correspondendo a uma cobertura de mais de $47 \%$ da população ${ }^{1}$. Tanto o M inistério da Saúde ${ }^{2}$ como a Sociedade Brasileira de Medicina de Família e Comunidade (SBM FC) ${ }^{3}$ consideram que a rede de serviços de atenção primária de saúde (APS) pode resolver $85 \%$ dos problemas de saúde da população. Contudo, para que haja tal resolubilidade, o nível secundário de atenção tem de assegurar 0 acesso dos usuários a consultas e exames especializados, indispensáveis para a conclusão de diagnósticos pela APS.

0 papel complementar dos diferentes níveis de atenção à saúde remete ao conceito da integralidade, entendida como a garantia do direito de acesso dos usuários às ações e serviços dos diferentes níveis decomplexidade, com fluxos ou percursos definidos e organizados espacialmente de forma a assegurar a continuidade dos cuidados em unidades localizadas o mais próximo possível dos cidadãos 4 . A integralidade da atenção numa rede de ações e serviços de saúde pressupõe, ainda, a correspondência entre a escala das unidades de aten ção, o território esua população. As unidades de cada nível de atenção são capazes desolucionar problemas de saúde deum determinado número de pessoas e devem ser dimensionadas de forma a garantirem essa oferta de serviços com qualidade. No PSF, definiu-se população mínima de 2.500 e máxima de 4.000 pessoas por equipe.

0 principal referencial para a abordagem da questão do atendimento integral à saúde, ou da integralidade da assistência no SUS, é fornecido pelos textos da Constituição Federal de 1988 eda Lei Orgânica da Saúde ${ }^{5}$. A primeira estabelece como segunda diretriz do Sistema Único de Saúde o "atendimento integral, com prioridade para as atividades preventivas, sem prejuízo dos serviços assistenciais" (art. 198, II). A Lei define como um dos princípios do Sistema a integralidade de assistência, entendida como conjunto articulado e contínuo das ações e serviços preventivos e curativos, indi viduais e coletivos, exi gi dos para cada caso em todos os níveis de complexidade do sistema (art 70, inciso II). 0 recentePacto pelaSaúde ${ }^{6}$, proposto pelo ministério, adota as mesmas definições.

0 principal elemento para a integração das redes desaúde éum efetivo sistema de referência e contrarreferência (RCR), entendido como mecanismo de encaminhamento mútuo de pacientes entreos diferentes níveis de complexidadedos serviços. O M inistério da Saúde define este sistema, inclusive, como um dos elementos-chave de reorganização das práticas de trabalho que devem ser garantidas pelas equipes de saúde da família (ESF)?

A abordagem da integração dos sistemas e redes de saúde envolve diversos aspectos interrelacionados, tais como: regulação dos serviços; processos de gestão clínica; condições de acesso aos serviços; recursos humanos; sistemas de informação e comunicação eapoio logístico. Diversos desses aspectos são considerados por Hartz e Contrandiopoulos ${ }^{8}$ como elementos críticos para se garantir a integração das redes de saúde, sendo quealguns deles constam como princípios orientadores do processo de regulação da atenção estabelecidos pelo Pacto pela Saúde ${ }^{6}$, de 2006. Podeseconsiderá-los como condições necessáriastambém para o bom funcionamento do sistema de referênciaecontrarreferência.

A regulação dos serviços é tarefa indelegável do gestor do sistema de saúde e envolve processos tais como: planejamento da oferta de ações e serviços com base nas necessidades de saúde da população; estabelecimento de responsabilidades e de metas quantitativas e qualitativas da atenção para as unidades de prestação de serviços dos diferentes níveis de complexidade; regulação da utilização dos serviços; monitoramento e avaliação dos resultados alcançados para a correção dos processos de trabalho.

Quanto à gestão clínica, não há um conceito claramente estabelecido a este respeito. Segundo Wagner ${ }^{9}$, usa-se o termo gestão de doenças, ou disease management, para designar o trabalho de coordenação de equipes multiprofissionais de cuidados que lidam com pacientes portadores de doenças crônicas. $M$ as trata-se de termo de abrangência mais limitada, voltado especificamente para o manejo integrado de pacientes crônicos. 0 conceito degestão clínica éinspirado em Eugênio V. M endes e utilizado por $\mathrm{H}$ artz e Contrandiopoulos ${ }^{8}$ para designar a aplicação de "tecnologias de microgestão" necessárias para meIhorar a qualidade e a eficiência dos serviços de saúde. Envolve aspectos como protocolos clíni$\cos$ (inclusivede RCR); educação permanenteem 
serviço; regulação das filas de espera para consultas, exames diagnósticos e internação; e supervisão ou apoio técnico para as equipes de ponta. Sua desconsideração pode prejudicar a implantação dos sistemas de referência e contrarreferência.

As condições deacesso da população às ações e serviços de saúde dependem tanto de sua proximidade das unidades dos diferentes níveis de complexidadequanto do dimensionamento adequado da oferta em relação ao número de habitantes e suas necessidades de saúde. Um elemento crítico para a garantia do acesso éo direito da população à informação sobre os serviços disponíveis, seus respectivos horários e as precondições requeridas para 0 acesso aos procedimentos especializados. Isto demanda, inclusive, uma denominação clara de cada nível de atenção e das unidades e serviços ofertados a fim de facilitar sua identificação pelos usuários.

Em relação aos recursos humanos, é necessário considerar a formação e a experiência profissional, as condições de trabalho e a existência de mecanismos de educação permanente, para assegurar a atualização dos seus conhecimentos. No Brasil, a formação para a saúde da família é ainda recente e insuficiente em termos quantitativos e as condições de trabal ho não são as ideais, incluindo salários baixos evínculos empregatícios precários, além de mecanismos de educação permanente ainda pouco desenvolvidos.

A regulação adequada de uma rede de ações eserviços de saúde requer quese conte com apoio de sistema de informações voltado para a identificação dos pacientes (como o Cartão SUS); 0 acesso a prontuários eletrônicos pelos profissionais das diferentes unidades; o controle da disponibilidade de leitos e vagas para consultas e exames; além do monitoramento das ações desenvolvidas. A inexistência ou o mau funcionamento de sistema de informação desse tipo dificulta 0 encaminhamento dos pacientes, seu acesso aos serviços, assim como a capacidade do gestor de controlar e avaliar o cumprimento dos objetivos e metas estabelecidos.

Da mesma forma, o apoio logístico eficienteé condição essencial para o bom funcionamento de uma rede de serviços de saúde, de forma a se garantir o abastecimento regular de medicamentos einsumos, inclusiveamostras deexames, além do transporte de pacientes. A manutenção e a calibração regular dos equipamentos biomédicos utilizados em todas as unidades é outro requisito logístico. A deficiência nesse setor pode resultar não só em desabastecimento como tam- bém em prejuízos para a qualidade do acompanhamento da situação dos pacientes.

A pesquisa encontrou problemas em relação a todos os aspectos por ela abordados. Os mais sérios dizem respeito à carência de planejamento da oferta e da regulação de vagas para procedimentos especializados, evidenciando, dessa forma, a ausência de elementos indispensáveis ao bom funcionamento do sistema de referência e contrarreferência, objeto central do estudo.

\section{Métodos}

0 desenvolvimento da pesquisa envolveu como métodos: análise crítica de fontes bibliográficas e documentais; levantamento de dados de caráter secundário sobre as redes de ações e serviços de saúde do SUS nas áreas pesquisadas; realização de entrevistas com amostra de médicos de família nas duas áreas abrangidas, com base em questionário semiestruturado. Os procedimentosutilizados foram de cunho predominantemente qualitativo, embora alguns dados tenham recebido tratamento quantitativo (estatística simples). Foram entrevistados 25 médicos de família, sendo 13 na AP 3.1 (Rio de Janeiro), de um total de 27, e12 em Duque de Caxias, de um total de trinta, correspondendo a $48 \%$ e $40 \%$, respectivamente, do universo de cada área. Em Duque de Caxias não foram considerados 37 médicos, cujas equipes ainda estavam em processo de implantação. A análise dos dados coletados por meio de roteiros de entrevista foi feita com base na metodologia de análise de conteúdo sistematizada por Bardin ${ }^{10}$.

\section{Considerações sobre o problema}

A partir do século XX, diversos fatores concorreram para aumentar os custos do setor de saúde, tornando a questão do acesso da população aos seus serviços e ações uma questão cada vez mais complexa. Os principais fatores que contribuíram para isto foram: a oferta de procedimentos de diagnóstico e terapia, associados a novas especialidades médicas e não médicas; a intensa incorporação tecnológica - equipamentos e fármacos; o aumento da longevidade da população, que vem ampliando a demanda por ações e serviços de saúde; e o crescimento das condições crônicas. 0 efeito combinado desses fatores tornou a questão da garantia do acesso um problema crucial para todos os sistemas de saúdee para 
a população, cuja solução depende cada vez mais do âmbito da política.

Segundo a $\mathrm{OM} \mathrm{S}^{11}$, a tradicional separação entre as doenças transmissíveis e as doen ças crônico-degenerativas vem perdendo importância. Isto deriva tanto da maior importância de novas formas de doenças transmissíveis - que afetaram até mesmo as populações dos países mais desenvolvidos, como o HIV/Aids e formas resistentes de tuberculose e sífilis - quanto do crescimento das tradicionais doenças crônico-degenerativas entre os países em desenvolvimento. Aquele organismo internacional vem defendendo, desde 2002, a adoção de um conceito mais amplo, denominado "condições crônicas", que envolve: doenças crônico-degenerativas; doenças transmissíveis de tratamento prolongado; problemas de saúde mental e sequelas de acidentes.

As "condições crônicas" representam, atualmente, a maior parte do aumento da carga de doença em todo o mundo. A OMS estima para 2020 que $80 \%$ da carga de doença dos países em desenvolvimento devem advir dessas condições. Os sistemas e serviços de saúde e a formação dos profissionais do setor, no entanto, sempre se voltaram para os casos agudos e não para as condições crônicas, queincluem, prioritariamente, ações deprevenção; promoção; detecção precoce; econtrole- tratamento continuado para evitar o agravamento dos casos. Essas condições exigem, portanto, a ação integrada de diferentes serviços e profissionais de todos os níveis de complexidade do sistema de saúde numa rede efetiva de serviços, com garantia de continuidade dos cuidados.

A maior parte dos sistemas de saúde existentes - inclusive o Sistema Ú nico de Saúde - está despreparada, no entanto, para dar conta dessa nova situação. Um dos elementos essenciais para que as ações e os serviços de saúde funcionem de forma integrada numa rede de serviços éo funcionamento de um sistema de referência e contrarreferência (RCR). No Brasil, inclusive, o M inisté rio da Saúde ${ }^{7}$ estabelecea referência econtrarreferência como um dos elementos-chave para as práticas de trabalho do Programa Saúde da Família (PSF). N esse sentido, a pesquisa realizada visou conhecer o funcionamento do sistema de referência e contrarreferência em duas áreas da Região M etropolitana do Rio de Janeiro (Área Programática 3.1, do Município do Rio de Janeiro, e o município de Duque de (axias), visando conhecer seus principais nós críticos, de forma a se obterem subsídios para a sua melhora no SUS.

\section{Resultadosediscussão}

A AP 3.1, com 872 mil habitantes ${ }^{12}$, está localizada na Zona Norte do município do Rio de Janeiro, faz limite com Duque de Caxias, que tem uma população de 855 mil pessoas ${ }^{13}$ e pertence à Baixada Fluminense. Estaárea programática 3.1 conta com uma Coordenação (CAP 3.1) eébem servida dehospitais do SUS (oito) eunidades secundárias (dez), tendo um número ainda relativamente limitado de unidades primárias (22), sendo 11 do PSF (27 equipes), além de uma unidade do PACS. Em Duque de Caxias há três hospitais, 12 unidades secundárias, noveunidades tradicionais deatenção primária e 67 equipes de saúdeda família em funcionamento, a maior parte delas (37) ainda em processo de implantação, resultando numa cobertura do programa superior a $30 \%$ da população. Na AP 3.1, ao contrário, há uma baixa cobertura do PSF (13\%), em virtude do lento processo de implantação do programa no município do Rio de Janeiro como um todo.

$\mathrm{Na}$ AP 3.1 há um esforço da SM S e da Coordenação da Área deorganização da rede deserviços, tendo sido definidos três subsistemas, cada um deles com um hospital geral e unidades de atenção secundária de referência. Para dar suporte às equipes de saúde da família, foi implantado um Grupo de Apoio Técnico (GAT), constituído por profissionais de saúde de diversas especialidades. A oferta de serviços de caráter secundário em apoio ao PSF não é planejada, nem está subordinada à CAP, dependendo de negociações entre esta e os dirigentes das unidades secundárias e terciárias, o que não assegura uma oferta regular e suficiente de procedimentos nesses níveis de atenção.

Em Duque de Caxias não há áreas ou subsistemas de saúde. Em 2006, foi implantado um sistema de regulação para consultas especializadas em apoio ao PSF, que, embora incipiente, vem melhorando o acesso a elas, segundo os médicos de família entrevistados. Não existia nada semelhante em relação aos exames diagnósticos, nem apoio técnico para as ESF.

Em ambas as áreas não houve planejamento da oferta de consultas e exames especializados com base nas necessidades de saúde da população. A pesquisa verificou um grau relativamente baixo de controle dos gestores dos sistemas sobre a oferta de serviços pel as unidades secundárias ou de média complexidade, cujas chefias são nomeadas politicamente. 0 baixo nível de regulação da rede encontrado nas duas áreas resulta em dificuldades para o encaminhamento dos 
pacientes do PSF para consultas e exames especializados, de acordo com as informações prestadas pelos médicos de saúde da família entrevistados (tabelas 1 e 2).

$\mathrm{N}$ as duas áreas, apenas sete tipos de consultas especializadas representam dois terços das dificuldades relatadas pelos médicos do PSF entrevistados. Algumas delas são críticas em relação às prioridades do programa, tais como: cardiologia (16,5\% das respostas na AP 3.1 e 6,5\% em Duque de Caxias), o que dificulta o controle adequado dos hipertensos; ginecologia $(5,6 \%$ na AP 3.1); e endocrinologia (6,5\% em Duque de Caxias). Também há nas duas áreas pesquisadas dificuldades para a referência para outras especialidades, como angiologia, neurologia, oftalmologia e ortopedia.

Os sete exames mais citados pelos médicos de família como os de maior dificuldade para referência representam $88 \%$ dos problemas na AP 3.1 e 70,3\% em Duque de Caxias, sendo que alguns deles - mamografia, dosagem hormonal, eletrocardiograma e ultrassom - são fundamentais para as prioridades do PSF.
Os principais problemasidentificados queinterferem no encaminhamento dos pacientes do PSF para o nível secundário de atenção são apresentados na Tabela 3, de acordo com as categorias de análise utilizadas. Três problemas se destacam em ambas as áreas: (1) inexistência ou precariedade da contrarreferência; (2) limitada oferta de consultas e exames; e (3) má organização das atividades de regulação. Outro problema mencionado que merece destaqueéa dificuldade de acesso dos pacientes aos serviços, por conta de dificuldades financeiras para arcarem com o custo das passagens, principalmente em Duque de Caxias.

Um achado eventual, mas importante, da pesquisa no que diz respeito à organização da redede saúdefoi a existência de nove denominações ediferentes configurações das unidades secundárias de saúde nas duas áreas, o que caracteriza uma aparente falta de critérios para a organização desse nível de atenção. Encontraram-se as seguintes denominações para elas: Centro de Atenção Psicossocial (CAPS); Centro deAtenção Psicossocial Infanto-Juvenil (CAPSIJ); Centro Especial deAten-

Tabela 1. AP 3.1 e Duque de Caxias, consultas especializadas do nível secundário com maiores dificuldades para referência.

\begin{tabular}{lccccccc}
\hline & AP 3.1 & & & \multicolumn{3}{c}{ Duque de Caxias } \\
\cline { 1 - 2 } \cline { 6 - 7 } Especialidade & $\%$ & $\%$ acum & & Especialidade & $\%$ & $\%$ acum. \\
\hline Cardiologia & 16,7 & 16,7 & & Angiologia & 16,1 & 16,1 \\
Neurologia & 13,9 & 30,6 & & N eurologia & 12,9 & 29,0 \\
Oftalmologia & 8,3 & 38,9 & & Oftalmologia & 9,7 & 38,7 \\
Ortopedia & 8,3 & 47,2 & & Proctologia & 9,7 & 48,4 \\
Otorrino & 8,3 & 55,6 & & Cardiologia & 6,5 & 54,8 \\
Angiologia & 5,6 & 61,1 & & Ortopedia & 6,5 & 61,3 \\
Ginecologia & 5,6 & 66,7 & & Endocrinologia & 6,5 & 67,7 \\
\hline
\end{tabular}

Fonte: informações prestadas pelos médicos entrevistados na pesquisa.

Tabela 2. AP 3.1 e Duque de Caxias, exames especializados com maiores dificuldades para referência.

\begin{tabular}{|c|c|c|c|c|c|}
\hline \multicolumn{3}{|c|}{ AP 3.1} & \multicolumn{3}{|c|}{ Duque de Caxias } \\
\hline Exame & $\%$ & $\%$ acum. & Exame & $\%$ & $\%$ acum. \\
\hline M amografia & 28,0 & 28,0 & Dos. hormonal & 21,6 & 21,6 \\
\hline Eletrocardiograma & 20 & 48,0 & Ultrassom & 13,5 & 35,1 \\
\hline Ultrassom & 12,0 & 60,0 & Tomografia & 10,8 & 45,9 \\
\hline Radiografia & 12,0 & 72,0 & HIV & 8,1 & 54,1 \\
\hline Ecocardiograma & 8,0 & 80 & Eletrocardiograma & 5,4 & 59,5 \\
\hline US de próstata & 4,0 & 84,0 & M amografia & 5,4 & 64,9 \\
\hline Dos. hormonal & 4,0 & 88,0 & Raios X & 5,4 & 70,3 \\
\hline
\end{tabular}

Fonte: informações prestadas pelos médicos entrevistados na pesquisa. 
Tabela 3. Maiores problemas para as referências, na opinião dos médicos.

\begin{tabular}{lrc}
\multicolumn{1}{c}{ Categoria } & AP 3.1 & Duque de Caxias \\
\hline Acesso & 5,3 & 22,2 \\
Comunicação & 2,6 & 5,6 \\
Contrarreferência & 21,1 & 16,7 \\
Gestão clínica & 5,3 & - \\
Logística & 2,6 & - \\
Oferta limitada & 42,1 & 22,2 \\
Regulação & 21,1 & 33,3 \\
Total & 100,0 & 100,0 \\
\hline
\end{tabular}

Fonte: informações prestadas pelos médicos entrevistados na pesquisa.

ção aosPortadores de Deficiência(CEAPD); Centro Especial deAtenção Total ao Adolescente(CEATA); Centro M unicipal deSaúde (CM S); Policlínica; Posto de Assistência M édica (PAM ); Posto Municipal de Saúde (PMS); Posto de Urgência (PU). Quanto à configuração da grade de serviços ofertados, encontrou-se, em Duque de Caxias, uma variação de oferta de serviços especializados entre 13 e 20 tipos para unidades com a mesma característica e denominação (PMS). Tamanha variação pode dificultar tanto o encaminhamento por parte dos profissionais quanto a identificação, pelos usuários, dos serviços que podem atender às suas necessidades.

Em relação aos aspectos relacionados à gestão clínica investigados pela pesquisa, 92,9\% dos entrevistados na AP 3.1 declararam que utilizavam protocolos para o encaminhamento para consultas especializadas, enquanto apenas $41,7 \%$ dos entrevistados disseram o mesmo em Duque de Caxias. As diferenças entre as duas áreas são ainda maiores em relação à utilização de protocolos para o encaminhamento de exames: na AP 3.1 , novamente $92,9 \%$; enquanto em Duque de Caxias apenas $16,7 \%$ dos médicos disseram utilizá-los. A contrarreferência nunca ou quasenunca ocorre nas duas áreas pesquisadas, segundo a maioria dos entrevistados $(85,7 \%$ das respostas na AP 3.1; e 91,7\% em Duque de Caxias), apesar de ser fundamental para a garantia da continuidade dos cuidados e o controle da situação de saúde dos pacientes acompanhados pelo PSF.

Ainda em relação à gestão clínica, encontrouse uma significativa diferença entre as duas áreas quanto à existência dos grupos de apoio técnico para os profissionais do PSF. Enquanto na AP $3.185,7 \%$ dos médicos disseram contar com apoio técnico, apenas $33,3 \%$ dos médicos de Duque de Caxias informaram o mesmo. Embora haja equipe deapoio técnico naAP 3.1, o número dos seus integrantes e a variedade de especialistas foram considerados insuficientes pelos médicos entrevistados. 0 apoio técnico existente em Duque de Caxias refere-se apenas aos programas verticais de saúde.

Em Duque de Caxias, por não ter havido organização territorial da rede, observaram-se sé rios problemas de acesso da população aos serviços secundários; as unidades de referência em Duque de Caxias estão em geral localizadas no Centro da cidade, queémuito distanteparagrande parte dos usuários. Outros fatores que prejudicam 0 acesso em ambas as áreas, segundo os entrevistados, são a violência - muitos usuários não podem sair cedo de casa por conta da dominação de seus locais de moradia pelo crimeorganizado - e dificuldades financeiras que impedem muitos de pagarem as passagen s necessárias para se deslocarem até as unidades de referência. Os maiores problemas em relação ao acesso a consultas e exames especializados, entretanto, decorrem das deficiências de gestão, como a falta de planejamento da sua oferta e a pequena capacidade de regulação por parte dos gestores.

Quanto aos recursos humanos, a pesquisa revelou situações distintas nas duas áreas pesquisadas em relação ao vínculo funcional dos médicos de família. Na AP 3.1, apenas $21,4 \%$ dos médicos de família entrevistados declararam ser funcionários públicos de carreira, enquanto em Caxias essa proporção era de $91,7 \%$. $N$ as duas áreas pesquisadas, verificou-seuma predominância de pediatras entre os médicos de família (50,0\% na AP 3.1 e 41,7\% em Duque de Caxias), seguidos pelos gineco-obstetras e clínicos gerais. Uma proporção relativamente alta entre esses profissionais não recebeu capacitação específica para a função (64,3\% na AP 3.1 e 33,3\% em Duque de Caxias).

Com relação à disponibilidade de sistemas de informação de apoio à gestão e regulação da rede, nenhuma das duas áreas tinha implantado - Cartão SUS ou contava com prontuários ele trônicos e sistemas eletrônicos de marcação de consultas e exames especializados. $\mathrm{Na}$ Ap 3.1, todas as USF dispunham de telefones e a grande maioria $(92,9 \%)$ de computador, embora apenas 7,1\% destes estivessem ligados em rede com a CAP. Em Duque de Caxias, apenas 8,3\% das unidades contavam com os dois meios de comunicação; as demais ficavam praticamente isoladas da rede SUS e da própria coordenação do 
programa. Tal precariedade em termos de informação ecomunicação prejudica o funcionamento do sistema de referência e contrarreferência nas duas áreas.

Em termos do apoio logístico às USF, os médicos de família entrevistados relataram ser frequente a falta de medicamentos nas duas áreas pesquisadas, sendo o problema ainda pior na AP 3.1 ( $85,7 \%$ das respostas) do que em Duque de Caxias (66,7\%), como mostram os dados da Tabela 4. Este problema compromete a proposta de controle de doenças queo PSF devefazer, particularmente em relação à hipertensão e ao diabetes, cujas prevalências são elevadas, o que ge ralmente redunda em intercorrências graves e de alto custo.

Também se constatou que praticamente não há manutenção e calibração dos equipamentos biomédicos utilizados pelo PSF. As respostas negativas dos médicos a este quesito foram elevadas nas duas áreas (71,4\% na AP 3.1 e 83,3\% em Duque de Caxias). A inexistência demanutenção e calibração dos equipamentos pode induzir a graves erros de diagnósticos e prescrições indevidas, com sérias consequências para os usuários.

\section{Consideraçõesfinais}

A pesquisa revelou a existência de deficiências no que diz respeito às condições operacionais necessárias ao bom funcionamento do sistema de re ferência e contrarreferência entre a saúde da família e os demais níveis de complexidade. Tais deficiências foram encontradas em todos os aspectos considerados: planejamento e regulação; gestão clínica; acesso aos serviços; recursos humanos; sistemas de informação e comunicação; além de apoio logístico.

$\mathrm{N}$ as duas áreas analisadas, os órgãos municipais degestão do SUS não detêm comando efetivo sobre as unidades de saúde secundárias em

Tabela 4. Frequência da falta de medicamentos.

\begin{tabular}{lrc}
\hline Área/frequência & AP 3.1 & Duque de Caxias \\
\hline Frequentemente & 85,7 & 66,7 \\
Raramente & 7,1 & 25,0 \\
Nunca & 7,1 & 8,3 \\
Total & 100,0 & 100,0
\end{tabular}

Fonte: informações prestadas pelos médicos entrevistados na pesquisa. razão das suas dificuldades em termos de planejamento e regulação e da indicação política dos dirigentes das unidades de saúde. A oferta das ações e serviços de saúde continua voltada para 0 atendimento da demanda espontânea e não para as necessidades de saúde da população, com base em critérios epidemiológicos, como o estabelecido pela Lei $n^{\circ} 8.080 / 90$ (art. 7ㅇ, VII). A distribuição espacial al eatória das unidades de saúdeeseu dimensionamento inadequado à demanda também são reflexos da falta de planejamento.

Em relação à gestão clínica, verificou-se a carência ou utilização deficiente de protocolos clínicos, cuja existência praticamente se restringe aos programas verticais de controle de doenças do M inistério da Saúde, tais como os programas de controle da hipertensão, diabetes, hanseníase, tuberculose. Os encaminhamentos para os serviços de referência são pouco apoiados nas diretrizes e nos protocolos clínicos existentes, e os médicos nem sempre os consideram plenamente. Isto revela não só a necessidade de melhor treinamento através de programas de educação permanente como também a importância do envolvimento desses profissionais na adaptação das diretrizes e dos protocolos à realidade local.

A deficiência do apoio técnico às equipes de saúde da família foi constatada nas duas áreas, 0 que dificulta a tomada de decisão clínica por seus profissionais, principalmente nos casos que estão no limite de seus conhecimentos, o que pode contribuir para aumentar o número de encaminhamentos para os demais níveis de complexidade. A contrarreferência se revelou praticamente inexistente nas áreas estudades.

Os problemas de acesso da população aos serviços não parecem ter sido considerados com a devida importância nas áreas analisadas. Distâncias exageradas ou dificuldades de pagamento das tarifas de transporte público criam dificuldades de acesso aos serviços pela população. A identificação dos serviços secundários ofertados é muitas vezes dificultada pela variedade de nomenclatura dasunidades secundárias (há nove denominações diferentes) e pela grande diversidade das grades de serviços oferecidos. A má organização dos serviços de exames diagnósticos exige, por vezes, o comparecimento dosusuários muito cedo, apesar de muitos terem dificuldades por conta da violência. Tais dificuldades podem ser superadas por meio de planejamento cuidadoso e de providências relativamente simples, como a implantação da coleta de exames laboratoriais nas USF e a implantação de agendamento para exames de imagem ou métodos gráficos. 
As principais deficiências quanto aos recursos humanos encontradas foram: profissionais sem formação específica para a saúde da família; pouco treinamento inicial para o exercício dafunção; esquemas precários de educação permanente; vínculos funcionais irregulares; grande isolamento dos profissionais, que trabalham em pequenas unidades e sem apoio técnico adequado.

Ossistemas deinformação ecomunicação existentes são precários. M uitas equipes não contam com telefones, nem computadores em rede. $N$ ão há prontuários eletrônicos, nem sistemas deagendamento de consultas e exames. 0 meio mais utilizado para a referência e contrarreferência é através de formulários em papel. Em relação ao apoio logístico, a pesquisa en controu uma falta generalizada e frequente de medicamentos e insumos, 0 que prejudica a eficária do controle de doenças prioritárias para o PSF (como hipertensão e diabetes). Os equipamentos disponíveis nas unidades não contam com manutenção adequada nem calibração, tornando-se pouco confiáveis.

\section{Referências}

1. Brasil. M inistério da Saúde. Departamento de Atenção Básica. Evolução do credenciamento e implantação da Estratégia Saúde da Família, 2008. [acessado 2008 jul 14]. Disponível em: http://dtr2004. saude.gov.br/dab/localiza_cadastro.php

2. Brasil. Ministério da Saúde. Assessoria de Imprensa. Programa Saúde da Família chega a mais 219 municípios; 2002 mar 19. [acessado 2007 jul 30]. Disponível em: http://portal.saude.gov.br/portal/ aplicacoes/noticias

3. Anderson MIP, Demarzo M, Rodrigues RD. A medicina de família e comunidade, a atenção primária à saúde e o ensino de graduação: recomendações e potencialidades. Rio de Janeiro: Sociedade Brasileira de Medicina de Família e Comunidade; 2007. [acessado 2007 jul 30]. Disponível em: http:/ /www.sbmfc.org.br

4. Giovanella L, Lobato LVC, Carvalho AI, Conill EM, Cunha EMDA. Sistemas municipais de saúde e a diretriz da integralidade da atenção: critérios para a avaliação. Saúde em Debate 2003; 26(60):37-61.

5. Brasil. Lei $n^{\circ} 8.080$, de 19 de setembro de 1990. Dispõe sobre as condições para a promoção, proteção e recuperação da saúde, a organização e o funcionamento dos serviços correspondentes e dá outras providências. Diário Oficial da União 1990; 20 set.

6. Brasil. M inistério da Saúde. Portaria GM /M S no 399. Divulga o Pacto pela Saúde 2006: consolidação do SUS e aprova as diretrizes operacionais do referido pacto. Diário Oficial da União 2006; 22 fev.
A situação encontrada, em síntese, contribui para uma menor resolubilidade do programa, provavelmente acarretando 0 agravamento desnecessário das condições de saúde de diversos pacientes, piorando sua qualidade de vida, além de sobrecarregar as unidades de maior complexidade do SUS. Nessas condições, o funcionamento do sistema de referência econtrarreferência fica prejudicado, assim como a necessária integralidade e continuidade dos cuidados.

\section{Colaboradores}

CG SerraePHA Rodriguespartciparam, igualmente, de todas as etapas de elaboração do artigo.

7. Brasil. Ministério da Saúde. Secretaria de Assistência à Saúde. Coordenação de Saúde da Comunidade. Saúde da Família: uma estratégia para a reorientação do modelo assistencial. Brasília: Ministério da Saúde; 1997.

8. Hartz ZM A, Contandriopoulos AP. Integralidade da atenção e integração de serviços de saúde: desafios para avaliar a implantação de um "sistema sem muros". Cad Saude Publica 2004; 20(Suppl.2):S331-S336.

9. Wagner $\mathrm{EH}$. The role of patient care teams in chronic disease management. BMJ 2000; 26:569-572.

10. Bardin L. Análise de conteúdo. Lisboa: Edições 70; 2004.

11. Organização Mundial da Saúde. Cuidados inovadores para condições crônicas: componentes estruturais de ação: relatório mundial. Brasília: OM S; 2003.

12. Rio de Janeiro. Secretaria Municipal de Saúde. AP 3.1 Através de mapas [CD-ROM]. Rio de Janeiro: Secretaria M unicipal de Saúde, Coordenação da Área Programática 3.1; 2006.

13. Brasil. M inistério da Saúde. Departamento de Informática do SUS. Informações de saúde, demográficas e socioeconômicas. [site da Internet] [acessado 2007 maio 15]. Disponível em: www.datasus.gov.br

Artigo apresentado em 16/11/2007

Aprovado em 27/06/2008

Versão final apresentada em 06/08/2008 